\title{
LAS RELACIONES DEL INTERNACIONALISMO CIENTÍFICO CON LOS ORÍGENES DEL MOVIMIENTO DOCUMENTAL EUROPEO CONTEMPORÁNEO (1890-1945)
}

\author{
Guillermo Olagüe de Ros* \\ Departamento de Historia de la Ciencia. Facultad de Medicina. Universidad de Granada.
}

\begin{abstract}
Resumen: En los orígenes del movimiento documental europeo contemporáneo el internacionalismo científico jugó un papel determinante. En este artículo se estudian los componentes teóricos, sociales y técnicos de dicho internacionalismo científico que contribuyeron al surgimiento de una naciente ciencia documental. Tras la I Guerra Mundial, el internacionalismo científico perdió su razón de ser, pero algunos de sus elementos siguieron teniendo un peso muy relevante en la evolución de la documentación científica.

Palabras clave: historia de la documentación científica; internacionalismo científico; movimiento documental europeo contemporáneo; Europa; siglos XIX-XX.
\end{abstract}

Title: THE RELATIONS OF THE SCIENTIFIC INTERNATIONALISM WITH THE ORIGINS OF THE EUROPEAN CONTEMPORARY DOCUMENTAL MOVEMENT (1890-1945).

Abstract: Scientific Internationalism played a determinant role in the beginning of the European contemporary documental movement. In this paper there are studied the theoretical, social and technical elements of the same one that contributed to the emergence of a nascent documentary science. After First World War, scientific internationalism lost his raison d'être, but some of his components continued having a very relevant weight in the evolution of scientific documentation.

Keywords: history of scientific documentation; scientific internationalism; European contemporary documental movement; Europe; 19th and 20th centuries.

\section{INTRODUCCIÓN}

En 1902, Torstein Jahr, asistente en la División de Catálogos en la Biblioteca del Congreso de los Estados Unidos, y Adam Julius Strohm (1870-1951), de la Biblioteca Pública de Trenton, publicaron una extensa relación de bibliografías surgidas entre 1850 y 1902 como resultas de una cooperación, nacional o internacional. En total dieron cuenta de 366 obras, lo que da la friolera cifra de más de siete publicaciones anuales sobre la cuestión. Es más, salvo cien todas se habían publicado desde 1890 (22’1 por año). La relación comprendía desde modestos repertorios hasta obras de gran envergadura, como la Bibliographie der Deutschen Naturwissenschaftlichen Literatur, cuyo primer volumen, publicado en abril de 1902, sobrepasaba las mil páginas, el International Catalogue of

*golague@ugr.es

Recibido: 06/05/2011; 2 a versión: 04/07/2011; 3ª versión: 23/02/2012; aceptado: 10/05/2012.

OLAGÜE DE ROS, G. Las relaciones del internacionalismo científico con los orígenes del movimiento documental europeo contemporáneo (1890-1945). Anales de Documentación, 2012, vol. 15, n 2. ISSN: 16977904. http://dx.doi.org/10.6018/analesdoc.15.2.126421. 
Scientific Literature (ICSL), auspiciado por la Royal Society de Londres, y el Répertoire Bibliographique Universel de Paul Otlet y Henri Lafontaine (Jahr y Strohm, 1902).

Cooperación internacional, internacionalismo y universalismo, fueron términos sinónimos usados con profusión por los científicos de todas las ramas, bien europeos o americanos, hasta las puertas de la I Guerra Mundial (Salomon, 1971). Con su utilización querían recalcar el carácter extranacional de la ciencia, su independencia de los gobiernos, su autonomía como forma de conocimiento, y la defensa de unos valores que estaban más allá de las absurdas disputas que mantenían entre sí los políticos de sus respectivos países, incapaces de entender que esos principios eran intrínsecos a la propia ciencia (SchroederGudehus, 1973). Para algunos teóricos de la época, el internacionalismo era además la consecuencia natural del pragmatismo inherente a los científicos, que al tener necesidad de intercambiarse información encontraban en la cooperación universal el marco idóneo que daba cuenta de esa premisa (Crawford, 1992).

Una de las manifestaciones de ese internacionalismo fueron las diferentes empresas trasnacionales encaminadas a coordinar esfuerzos en el desarrollo de la investigación y a favorecer el intercambio de resultados (Olagüe de Ros et alia, 1997). La confección de catálogos de bibliografía corriente, como el ya mencionado ICSL, fue uno de los cimeros de dicha colaboración internacional. El Répertoire Bibliographique Universel (1895), ligado al Institut International de Bibliographie de Bruselas (IIB) de Paul Otlet (18681944) y Henri Lafontaine (1854-1943) fue la otra gran empresa internacionalista del momento (Rayward, 1975, p. 44-45). Otras muestras de ese universalismo fue la abundancia de congresos internacionales para unificar pesos y medidas, normalizar terminologías científicas, estandarizar técnicas y aparatos registradores, consensuar estadísticas médicas y fijar definitivamente husos horarios y meridianos (Somsen, 2008). Además de las reuniones, el internacionalismo recurrió a otros elementos para reforzarse, como el patrocinio de asociaciones supranacionales, tales son la Asociación Internacional de Academias (1889) y la Unión de Asociaciones Internacionales (1911) (Edwards, 2004). El internacionalismo facilitó también una mejora en los intercambios personales, especialmente en el mundo académico. Por ejemplo, entre 1905-1914 treinta investigadores alemanes y otros tantos norteamericanos trabajaron pensionados en Harvard y en varias universidades prusianas, respectivamente (Richards, 1994, p. 3).

En este artículo se analizan algunos de los elementos sociales y científicos que estuvieron presentes en el nacimiento y desarrollo del internacionalismo científico y cómo, tras la finalización de la I Guerra Mundial, buena parte de ellos dejaron de ser considerados por la comunidad internacional.

\section{LA UNIVERSALIDAD DEL MÉTODO CIENTÍFICO Y LA FILOSOFÍA POSITIVISTA DE AUGUSTE COMTE}

Uno de los elementos que alimentó la concepción universalista de la ciencia fue de orden epistemológico, atisbado ya en plena Revolución Científica pero que alcanzó 
madurez doctrinal en la segunda mitad de la centuria decimonónica (Somsen, 2008). Si el método científico era uno y válido para todas las ramas del saber, era lógico concluir que era universal y que sus cultivadores, en tanto que científicos, eran internacionales ${ }^{1}$. Su patria era la ciencia, la única garante del progreso y del bienestar humano, fin al que se dedicaron los sabios de esta época con ahínco y entusiasmo, además de algunos mecenas. El otro componente fue de naturaleza socio histórica. Augusto Comte (1798-1857) entre 1830 y 1842 publicó su célebre Cours de Philosophie Positive, una densa obra en seis volúmenes que en síntesis venía a proclamar el fin de la metafísica y de la teología y el triunfo de la ciencia sobre ellas. Con Comte la ciencia se convertía en un agente de transformación social y, al mismo tiempo, en una garantía del orden. Como diría el mismo Comte: "el progreso es el desarrollo del orden". Serán los científicos, pues, los que en la última etapa de la evolución de la humanidad, la positivista, gobernarán el mundo, sustituyendo a los filósofos y a los representantes de las distintas religiones. En el fondo, Comte fue políticamente bastante conservador y un claro defensor del orden burgués, además de misógino, aunque tuvo una idea de la historia francamente progresista, al entender que ésta evolucionaba de manera continua hacia el progreso social y moral de la humanidad, gracias a la ciencia positiva (Hjørland, 2005).

El tercer componente que sustentó el internacionalismo fue el progreso tecnológico, pues permitió acortar distancias e hizo posible que científicos de diferentes países pudieran juntarse con relativa asiduidad, como luego comentaré.

\section{EL DESARROLLO DEL ESPECIALISMO}

Que el científico se liberara del secular mecenazgo de la nobleza y del clero y se convirtiera en un profesional fue otro hito en este proceso. Profesionalizarse supuso convertirse en un empleado asalariado del Estado, que únicamente exigía a sus científicos logros a cambio de medios y de una forma de vida decente, no constreñida por prejuicios religiosos o metafísicos. En los países más industrializados esos logros se traducían en patentes y en el desarrollo de áreas de investigación claves en la pujanza industrial de las naciones, por ejemplo en física y química, en las que durante decenios Alemania tuvo una supremacía apabullante sobre los demás Estados².

La profesionalización del científico abrió las puertas al especialismo. La fragmentación de la ciencia en ramas específicas del saber casaba mal con su sentido universalista, que era una de sus notas más señaladas, aunque fomentaba la edición de repertorios especializados en las diferentes ramas de la ciencia. Por ello, en aras de mantener cierto nivel de integración surgieron asociaciones, como el Bureau de la Fondation pour l'Internationalisme, que además de tener una actividad política muy intensa, editó algunas publicaciones integradoras, al menos en las grandes ramas de la ciencia (Eijkman, 1910 y 1911).

Por otro lado, el especialismo fue un estímulo para otras ramas del saber, no sólo en ciencia. En efecto, algunos bibliotecarios sintieron también la necesidad de especializarse 
para así cumplir mejor su labor informativa a los científicos. Este motivo fue la razón del surgimiento de los "bibliotecarios especializados" en el mundo anglosajón (special librarians), una experiencia que no se dio en Europa, salvo individualidades muy concretas (Farkas-Conn, 1990). Más bien, en el Viejo Continente la resistencia de los bibliotecarios más clásicos fue motivo de acres polémicas con los prodocumentalistas, como en Italia (Giunchedi y Grignani, 1994), y también en España (Olagüe de Ros et al., 1994).

\section{CIENCIA Y PACIFISMO}

Algunos intelectuales defensores de la filosofía comtiana, llevaron su firme creencia en el carácter universal y benévolo de la ciencia a otras facetas, algunas ligadas a esas notas, pero otras no tanto. Si para el positivismo el "conocimiento" era el bondadoso agente que a través del progreso iba a salvar a la humanidad, las obras impresas, monografías o revistas, se transformaban en la clave de esa salvación al ser las encargadas de preservar y difundir el saber. Bibliófilos, bibliotecarios y los primerizos documentalistas seguidores de Comte, abrazaron efusivamente el internacionalismo, y se convirtieron en convencidos defensores de la benevolencia del ser humano. No fue extraño, pues, ver a documentalistas y bibliotecarios comprometerse intensamente con los movimientos pacifistas, especialmente en los años previos y durante el curso de la I Guerra Mundial. La culminación de esa actitud fue la fundación de la Sociedad de Naciones (1920), en la que tan intensamente intervino Henri Lafontaine, que entre 1907 y 1943 presidió el Permanent International Bureau of Peace (1891) y por cuyos desvelos por la paz recibiría el Premio Nobel en 1913. Otro activo pacifista fue Herbert Haviland Field (1868-1921) un zoólogo americano residente en Zurich, fundador del Concilium Bibliographicum, un instituto de documentación bibliográfica en ciencias naturales, que también intervino activamente para impedir que Alemania iniciara las hostilidades (Ward, 1921). Field editó en su Instituto zuriqués algunas publicaciones defensoras de la paz, como las de Harold Fowler MacCormick (1872-1941), un acaudalado financiero americano, presidente de la Fundación Rockefeller, del que dio a luz sendos textos en 1916 y 1917.

\subsection{Mecenazgo y pacifismo en las grandes fortunas mundiales}

Los aires del internacionalismo y del pacifismo también alcanzaron a buena parte de las grandes fortunas mundiales, especialmente del mundo americano, que en parte se volcaron en políticas activas probibliotecarias. John Davison Rockefeller (1839-1937), que llegó a monopolizar las compañías petrolíferas de su país, creó en 1913 la Rockefeller Foundation, que desde su creación ha apoyado sustancialmente programas en el campo de la salud pública y de la prevención de la enfermedad (Brown, 1979). Recientemente, Rodríguez Ocaña (2000) ha analizado el interesante papel que jugó la Rockefeller en España, entre 1919-1939, en la puesta en marcha de programas de salud pública en áreas rurales olvidadas y en la formación de técnicos especialistas. En el ámbito de las bibliotecas se distinguió tras la II Guerra Mundial, pues ayudó sustancialmente a 
recuperar y levantar buena parte de las grandes bibliotecas europeas que habían sido destruidas por el conflicto.

Andrew Carnegie (1835-1919), un magnate de la industria del acero en Estados Unidos, en 1901 vendió todos sus activos financieros para entregarse de lleno a la causa de la paz y de la cultura. En 1910 fundó el Carnegie Endowment for International Peace, que desde entonces ha estado muy presente en todos los conflictos bélicos, actuando como intermediario y produciendo informes muy bien documentados sobre los mismos. Además, Andrew Carnegie potenció de manera muy importante el sistema bibliotecario americano, pues creó y dotó casi dos mil bibliotecas a lo largo del país (Alderson, 1902). A una escala más modesta, y con apoyo oficial, recordaré la Xarxa de bibliotecas públicas creada por la Mancomunitat de Catalunya en 1915, con la pretensión de dotar a todos los municipios catalanes de más de seis mil habitantes de una de esas bibliotecas. La Escola Superior de Bibliotecàries, creada ese mismo año, tenía por objetivo formar profesionales que se hicieran cargo de la dirección de las mismas. Como es bien sabido, tras la Guerra Civil se cortó radicalmente esa política cultural, que tan inteligentemente había dirigido durante años Jordi Rubió i Balaguer (Rovira, 1984).

En 1846, con la fortuna que legó a los Estados Unidos el británico James Smithson (1765-1829) se fundaba la Smithsonian Institution, cuyo objetivo era "aumentar y difundir el conocimiento". Una de las facetas en las que más se destacó fue en el intercambio de publicaciones científicas, cuestión a la que todos los años dedicaba una memoria en la que se daba cuenta de las instituciones y los países con los que la Smithsonian había llegado a acuerdos en este sentido (International, 1904). Por otro lado, su biblioteca fue desde el principio una de las instituciones más mimadas por los patrones de la fundación. Al frente de la misma estuvo entre 1892 y 1905 Cyrus Adler (1863-1941), que jugó un papel muy activo a favor de la participación de los Estados Unidos en el ICSL de la Royal Society, pues consiguió que la biblioteca de la Fundación fuera la oficina regional americana de dicho catálogo británico (Neumann, 1940-1941).

\section{LA LENGUA UNIVERSAL Y LAS LENGUAS AUXILIARES}

Para muchos científicos, la defensa de una lengua universal era un signo más del internacionalismo y un paso adelante en la desaparición de los estados nacionales, sustituidos por un nuevo modelo de relaciones en el que un "gobierno planetario" traería riqueza y bienestar al mundo (Andrews, 1871, p. VIII-IX). Además, comunicarse en una lengua común facilitaría las relaciones profesionales. Las soluciones propuestas fueron varias y, básicamente, no tuvieron en consideración los idiomas naturales. Se pretendió "crear" una nueva lengua que hermanara a la humanidad y, por tanto, a los científicos. La ristra de alternativas fue amplísima, pero de todas ellas el esperanto, ideado por el médico polaco Ludwik Zamenhoff (1859-1917) en 1887, fue la que más adeptos encontró (Guérard, 1921, p. 216-219). Además, la adopción de una lengua auxiliar universal encajaba perfectamente con los intentos de universalización de los lenguajes documentales de indización, de los que la CDU, bien en la versión de Melvil Dewey o en 
la modificada por Paul Otlet y Henri Lafontaine, o el sistema propuesto por la Royal Society, eran dos buenas iniciativas. Finalmente, una lengua universal acabaría con el lamentable espectáculo de la exclusión de ciertos idiomas, como el español, de los congresos internacionales de la época.

\section{RACIONALIDAD Y ORGANIZACIÓN CIENTÍFICA DEL TRABAJO}

Pero en el mundo de la ciencia la aceptación universal del internacionalismo y de las mejoras tecnológicas, aun siendo causas determinantes, no fueron las únicas que explican el empuje que la ciencia documental conoció en esas fechas. Había que organizar científicamente el trabajo al objeto de alcanzar la máxima rentabilidad, y en esa faceta el impacto del taylorismo fue determinante.

En 1912 Frederick Winslow Taylor (1856-1915) publicó un libro que revolucionó el mundo del trabajo, Principles of Scientific Management, el más acabado estudio hasta entonces sobre la organización racional del trabajo. Su tesis era que la máxima rentabilidad en la producción de bienes se podía conseguir en el mínimo tiempo especializando a cada obrero en una tarea específica. La suma de las tareas de todos los que participaban en la cadena productiva daba como resultas un producto completamente acabado, competitivo y listo para su consumo (Chanaron y Perrin, 1987). Una variante del taylorismo, que es como se conoció desde entonces la propuesta de este ingeniero americano, la aplicó Henry Ford en su cadena de montaje de coches de Detroit. Fue, precisamente, el fordismo el que tan ácidamente criticó Charles Chaplin en Tiempos Modernos (1936).

La tesis de Taylor, sustentada en el positivismo comtiano, fue muy bien recibida por los incipientes documentalistas, pues comprendieron que podían aplicar su idea de máxima rentabilidad con el mínimo esfuerzo en el proceso de recuperar y proporcionar la información científica que se les demandara en un tiempo ínfimo. Si la documentación consistía en la reunión, clasificación y distribución de documentos (Shera, Ceveland, 1977, p. 251), tan sólo una eficiente organización del trabajo podría facilitar al usuario la información óptima en el mínimo tiempo posible.

\subsection{Wilhem Ostwald (1853-1932) y Die Brücke (El Puente) (1911)}

En la segunda década del siglo XX, Wilhelm Ostwald (1853-1932), un químico alemán que recibiría el Premio Nobel en 1909, fue el primero en considerar las tesis tayloristas en el ámbito de la documentación científica (Burchardt, 1977). En 1911 fundó, con el dinero concedido por el Premio Nobel y tras renunciar en 1906 a su cátedra de Química Física en la Universidad de Leipzig, Die Brücke, Internationales Institut zur Organisierung der geistigen Arbeit. Adelantándose a Herbert George Wells (1866-1946), Ostwald concibió El Puente como un "cerebro mundial" (Ostwald, 1912; Sachsse, 1998), cuyo objetivo era "dar respuesta, directa o indirecta a cualquier cuestión intelectual que se le formulara, de manera que el usuario fuera aconsejado sobre el lugar idóneo donde podía obtener suficiente información sobre su consulta" (Ostwald, 1913, p. 6) ${ }^{3}$. 
En esa necesidad de racionalizar el trabajo intelectual, Ostwald defendió la estandarización del papel en los libros impresos -a él se debe la invención de la hoja Dina4- y la conveniencia de que algunos lo fueran con hojas sustituibles, de tal forma que los nuevos adelantos vendrían a reemplazar a las hojas obsoletas (Hapke, 1999, 2003 y 2004). Otro de los grandes logros de Ostwald fue su visión de la necesidad de los documentalistas científicos, los únicos capaces de cumplir con el principio taylorista de la organización científica y racional del trabajo (Ostwald, 1909, p. 175).

Finalmente, por razones pragmáticas, Ostwald y sus colaboradores de El Puente fueron importantes propagadores de la CDU de Melvil Dewey en el mundo alemán. K. W. Bührer y Adolf Saager, en un folleto editado por El Puente en 1912, precisamente acerca de la CDU como sistema de registro, la consideraron idónea, pues reunía siete características notables: "era científica y lógica en su fundamento; económica desde el punto de vista del gasto energético y práctica por su aplicación; internacional en cuanto a su consideración editorial; enciclopédica e ilimitada en su expansión; sistemática y metódica en el orden. Al mismo tiempo, era más exhaustiva en las grandes colecciones y más sintetizadora en las pequeñas; y, finalmente, era enciclopédica y válida como sistema de registro para todas los campos del saber” (Bührer y Saager, 1912, portada).

Una culminación de ese internacionalismo fue el proyecto de Hermann Beck ( $n$. 1879), que no se llevó a cabo, consistente en que cada país debía elaborar su bibliografía nacional. Firme partidario de la racionalidad en el trabajo intelectual, Beck fundó en 1908 el Institut für Techno-Bibliographie, que se especializó en organizar y resumir toda la literatura técnica de su tiempo. Una oficina central, en colaboración con bibliotecas y editoriales comerciales, se encargaría de confeccionar el repertorio universal, que se formularía sobre una división en grandes campos temáticos. El Instituto contaba con una gran biblioteca, una sección de información, un servicio de traducción, y una librería de venta de monografías al público (Beck, 1907).

\section{LOS ACUERDOS INTERNACIONALES Y LOS AVANCES TECNOLÓGICOS AL SERVICIO DE UNA DIFUSIÓN MÁS EFECTIVA DE LA INFORMACIÓN CIENTÍFICA}

A veces no valoramos suficientemente adelantos aparentemente nimios pero que han revolucionado el modo de trabajar en las bibliotecas. En el periodo que ahora me ocupa se dieron algunos avances que, especialmente en los países de mayor peso científico, supusieron cambios sustanciales en la dinámica y desarrollo documental. Uno de ellos fue el resultado de un acuerdo supranacional: La Unión Postal Internacional, creada en 1875. El otro fue de naturaleza técnica, la introducción del microfilm (Farkas-Conn, 1990, p. 1620).

La Unión Postal hizo que el correo fuera más eficiente y seguro. Los últimos adelantos, plasmados en fascículos de revista, llegaban con mejores garantías a las 
bibliotecas, cuyos responsables rápidamente los ponían en circulación para satisfacer a sus usuarios (Treaty, 1875).

El segundo avance, el microfilm, permitió facilitar copias de artículos y otros soportes documentales a estudiosos de otros lugares que carecían de los originales. Un signo de los tiempos es que el microfilm era una realidad desde hacía tiempo en el mundo de los negocios, pues fueron los bancos los primeros en recurrir al mismo para sus transacciones comerciales, aunque el primero que advirtió premonitoriamente su utilidad documental fue Paul Otlet, que en colaboración con Robert Goldschmidt, un ingeniero que cooperaba asiduamente con Otlet, publicaron en 1906 un folleto sobre la aplicación del microfilm en documentación (Richards, 1994, p. 14).

Entre mayo y noviembre de 1937 se celebró en París la Exposición Internacional bajo el lema "Artes y Técnicas de la vida moderna". El Gobierno de la República española participó con un Pabellón que contó con dos novedades francamente singulares: el Guernica de Picasso y la fuente de mercurio del norteamericano Alexander Calder (18981976). 1937 fue, como indicó Rayward, el último aliento de la "fe en el poder del intelecto, en la necesidad de la asociación internacional y en la inevitable interdependencia de la humanidad...”, pues todavía se pensaba que la civilización, finalmente, conseguiría superar las ancestrales e inoperantes rivalidades (Rayward, 1983, p. 254).

Julien Cain (1887-1974), administrador desde 1930 de la Bibliothèque Nationale, fue el encargado de ofrecer en el curso del Congrès Mondial de la Documentation Universelle, celebrado durante la Exposición International, y sobre el que más adelante volveré, una exposición con el pomposo rótulo de "Palacio de la Literatura, el Libro, las Artes Gráficas y las Artesanías”. En ella, y a través de cuatro secciones, Cain reprodujo en la primera una biblioteca actual, con sus estanterías, ficheros, mesas y sillas para los lectores. En la segunda, abordó el tema de las bibliotecas particulares, desplegando fotografías y planos en paneles desplazables. La tercera la dedicó a cuestiones profesionales, como la creación de catálogos, asociaciones profesionales y bibliotecas especializadas, como las hospitalarias. La última se centró en el equipamiento y medios técnicos al servicio de las bibliotecas. Cain fue de los bibliotecarios de estos años que mantuvo una actitud menos receptiva a la naciente documentación. Precisamente, en una intervención suya en el curso de ese Congreso se expresó muy claramente sobre la cuestión: “... la ciencia documental es una quimera. La documentación es una técnica, nada más” (Nicault, 2005).

La gran novedad de este "Palacio" fueron las dos secciones dedicadas a la microfotografía, una mostrando los recursos que ya se utilizaban en la Bibliothèque Nationale de París, y la otra dando cuenta de un laboratorio completo y dos cámaras fotográficas para microfilmación en uso en la Universidad de Chicago. No hay que olvidar que desde 1934 la National Library of Agriculture de los Estados Unidos había creado un Bibliofilm Service, el primero que recurrió al uso del microfilm en vez del tradicional 
préstamo interbibliotecario para publicaciones científicas (Rayward, 1983, p. 257). Para ello, adquirió la cámara ideada por el teniente de navío Rupert Draeger, que había sido utilizada exitosamente en la microfilmación de revistas de medicina que podían ser de utilidad en los barcos de guerra americanos (Richards, 1994, p. 14-15; Tate, 1950; Rayward, 1983).

El Congrès Mondial de la Documentation Universelle contó con el apoyo de la Sociedad de Naciones, acudieron casi quinientos participantes, y su cabeza visible fue Jean Gérard, presidente de la Union Française des Organismes de Documentation (Rayward, 1983; Jouot, 1997). Los asuntos que atrajeron la mayor atención de los participantes fueron el futuro uso del microfilm en las bibliotecas, y la charla inaugural que impartió Wells, en la cual, al referirse al microfilm, se expresó de esta forma: "El conocimiento no precisa tener la vulnerabilidad de una centralización material y física, pues las continuas mejorías de la fotografía hacen que la duplicación de nuestros índices y archivos sea cada vez más fácil y barata. En estos días de destrucción, violencia e inseguridad general, reconforta pensar que el cerebro de la humanidad, la raza humana, pueda pervivir a través de numerosas réplicas en todo el mundo, en Perú, Islandia, Gales y Nueva Zelanda, así como aquí mismo, en París” (cit. por Richards, 1994, p. 16).

Hasta tal punto entendió Wells que era trascendente el trabajo de los bibliotecarios y documentalistas que, según él, en los momentos de mayor declive de la sociedad serían los únicos capaces de salvaguardar la civilización. Los documentalistas proporcionarían, según Wells, el marco adecuado al Cerebro del Mundo (Rayward, 1999), es decir, la Gran Enciclopedia del Saber Mundial, una "potente estructura para la comparación, reconciliación y síntesis de las ideas comunes de toda la humanidad" y a la cual aquéllos estaban contribuyendo de manera importante (Rayward, 1983, p. 265).

Como mostró Rayward hace años, el congreso parisino de 1937, de escasas consecuencias para la ciencia documental, fue el escenario en el que afloraron distintos conflictos, como las rivalidades entre los dos sectores mayoritarios de la documentación del momento: el de Gérard, que pretendía organizar un sistema documental al margen de Bruselas, es decir, abogaba por un esquema federal y descentralizado de los servicios documentales, y el de Otlet y Lafontaine, más jerárquico y centralista, aunque mucho más extendido y numeroso, o el radical rechazo hacia la naciente ciencia documental, como el ya comentado de Cain (Rayward, 1975, p. 356-357). Sin embargo, el discurso inaugural de Wells y las novedades técnicas que en el mismo se presentaron fueron trascendentes y muy importantes ${ }^{4}$.

Un elemento que contribuyó de manera notable a una difusión más universal de las publicaciones científicas fue la política publicista del momento. En los países de tradición cultural germánica la fórmula habitual era el concierto entre las Editoriales y las Academias Científicas, que destinaban parte de la subvención estatal a este fin, con lo que se prestigiaban tanto la academia como la editorial comercial, que abría una vía en la especialización de sus publicaciones y aseguraba una difusión notable de las mismas. Esta 
opción garantizaba además un mercado estable y potente. Esta fórmula permitió el afianzamiento de grandes editoriales, como Elsevier, Springer Verlag etc. Por su parte, en las naciones de tradición inglesa imperó el modelo no concertado, es decir, las academias, instituciones científicas y fundaciones eran las editoras de sus propias publicaciones, sin intermediarios comerciales de por medio (Cook, 2003, p. 15-24).

\section{NUEVAS BIBLIOTECAS Y SU ORGANIZACIÓN RACIONAL ANTES DE LA GRAN GUERRA}

Las empresas reformistas en el ámbito cultural también alcanzaron a los lugares donde se preservaba el saber, las bibliotecas. En los primeros años del siglo XX se dio un interesante movimiento encaminado a hacer de los espacios de las bibliotecas templos laicos del conocimiento.

En 1905, por ejemplo, Middleton, vicepresidente de la londinense Sociedad de Arquitectos, editaba una muy completa obra en seis volúmenes con el título "Edificios Modernos. Su Planificación, Construcción y Equipamiento", para la que contó con un amplio abanico de colaboradores, Pues bien, en el último tomo se dedicaba la Sección Primera a "Edificios varios y sus accesorios", y en ella H. C. Querée abordaba el diseño arquitectónico y la dotación de recursos de una biblioteca moderna (Quérée, 1905, p. 5867).

También hay que destacar el papel desempeñado por Emil Pirchan (1884-1957) arquitecto y diseñador gráfico. Durante los años veinte y treinta fue muy requerido para diseñar carteles de propaganda de grandes empresas y de espectáculos teatrales de la época. Vinculado a Die Brücke de Ostwald fue el primero en preocuparse por la habitabilidad de los espacios bibliotecarios y por el boceto ergonómico de los lugares de trabajo. En colaboración con el también arquitecto e interiorista vienés Joseph Hoffmann (1870-1956), una de las personalidades ligada a la Bauhaus, planearon espacios y mobiliario ideales para bibliotecas (Bührer, 1912). Sus dibujos, muy sencillos en ejecución, muestran amplitud de volúmenes, luminosidad, y unos diseños para estanterías y puestos de trabajo funcionales y cómodos. Por su predilección por las líneas rectas fue llamado "Señor Cuadrado" (Sachsse, 1998).

Quizás el mejor exponente de esa nueva mirada hacia el mundo de las bibliotecas lo constituya la inauguración de la New York Public Library el 23 de mayo de 1911. Una vez más, el mecenazgo y la filantropía se dieron de la mano para lograr dotar a la ciudad de Nueva York de una de las más importantes bibliotecas del mundo. Jacob Astor (17631848), un inmigrante alemán que hizo fortuna en los Estados Unidos, donó una importante suma de su patrimonio personal para poner en marcha una biblioteca en la ciudad ${ }^{5}$. Por su parte, James Lenox había fundado otra biblioteca, mucho más especializada y repleta de libros de incalculable valor. En 1895 se fusionaron las bibliotecas de Lenox y la creada por Astor. Para dar cabida en un único espacio a los fondos de ambas se pensó en abrir una de nueva planta, que sería subvencionada con los fondos legados para tal fin por 
Samuel J. Tilden (1814-1886), antiguo gobernador del Estado, y se encargó a John Shaw Billings la ejecución y dirección de la misma. Personalmente diseñó la amplia sala de lectura y las siete plantas para el almacenaje de las colecciones presentes y futuras, e hizo que dos arquitectos noveles, Carrère y Hastings, dieran forma a su proyecto (Handbook, 1916).

\section{LA RETÓRICA DEL DISCURSO INTERNACIONALISTA}

El "internacionalismo científico" fue hasta cierto punto un discurso retórico que el propio curso de los acontecimientos en Europa vino a mostrar. El gran proyecto bibliográfico de principios de la centuria, el británico ICSL, que sintetizaba a la perfección todas las aspiraciones de dicha filosofía, como la relación entre iguales entre los diferentes países participantes en el mismo, en el fondo imponía dos condiciones que rompían con ese igualitarismo: una dirección jerárquica, la Royal Society de Londres, como oficina catalizadora de la información que le remitían las academias científicas de otros países, y una supremacía de algunos idiomas -fundamentalmente el inglés, el francés y el alemánsobre otros, quizás de menor peso en la comunidad científica internacional, pero más importantes por número de personas que lo hablaban, como el español (Lyons, 1968). Es bien conocido, por otra parte, que al estallar la I Guerra Mundial Otlet y Lafontaine abandonaron Bruselas y durante los años del conflicto se comprometieron intensamente con la causa de la paz mundial. En efecto, Lafontaine fue uno de los promotores de la Sociedad de Naciones (Rayward, 1975, p. 203-219) y por su parte Otlet tuvo un papel relevante en el curso inicial de la ONU (Sagredo, 2004).

Paradójicamente, el proyecto de la Royal Society sirvió también para reforzar la vertiente nacionalista de la ciencia de otros países. Es el caso de cinco bibliografías nacionales -Queensland (Australia) (1899), Francia (1901-1914), Rusia (1904-1917), Polonia (1901-1911) y Alemania (1901-1914)- nacidas de forma independiente como consecuencia de la participación de otros tantos países en el dicho repertorio. Tan interesantes iniciativas se interrumpieron a raíz de la I Guerra Mundial (Olagüe de Ros y Ignaciuk, 2011).

Al concluir la Gran Guerra, George Francis Hampson (1860-1936), Asistente del Museo de Historia Natural de Londres, publicaba en 1918 un artículo muy técnico sobre entomología en una revista de su especialidad. Al pie de la primera página del mismo incluía esta nota: "No se incluyen referencias de artículos de autores alemanes publicadas con posterioridad al uno de agosto de 1914". Y concluía con esta sentencia latina: "Hostes humani generis" ("enemigos del género humano") (Hampson, 1918, p. 55). Ese mismo año el alemán Fritz Haber (1868-1934) recibía el premio Nobel de Química por sus estudios sobre la síntesis del amoníaco, un producto básico en la lucha contra el hambre mundial. Paradójicamente, ese año era condenado por un Tribunal Internacional como criminal de guerra por el uso indiscriminado del gas clorina en las trincheras durante la I Guerra Mundial (Dubravsky, 2008). Finalmente, indicaré que en la conferencia Robert Solvay de Física de 1921, en la que se trató de "átomos y electrones", fueron excluidos los 
representantes alemanes (Mehra, 1975) ${ }^{6}$. Los científicos, pues, dejaban de ser internacionalistas, aunque la ciencia lo fuera. Desde ahora su objetivo sería contribuir a la creación de una ciencia nacional, propia, para lo cual el estado pondría los medios y recursos adecuados.

\section{CONCLUSIONES}

La vieja filosofía del internacionalismo, con sus premisas de hermandad y paz universal, había muerto hacia 1920. A partir de esa fecha, pues, la competencia técnica entre las naciones dominantes fue la tónica, y en esa batalla los países anglosajones tuvieron las de ganar, con más motivo después de 1945, cuando, de nuevo, Alemania y sus aliados salieron derrotados tras su enfrentamiento con el resto del mundo. Una consecuencia importante del predominio anglosajón fue el paulatino declive de las lenguas europeas continentales como idiomas de comunicación científica, y el creciente auge del inglés. La creación del repertorio Excerpta Medica es un claro ejemplo de este cambio de situación. Deseosos de enlazar con una tradición cultural arrasada por la guerra, Erich Landsberger y Janos Freud, dos médicos judíos alemanes que se librarían de la shoah, decidieron fundar antes de finalizar el conflicto un nuevo catálogo de resúmenes, al que bautizaron con el nombre de Excerpta Medica. La nueva Excerpta Medica, pues ya hubo otra en el siglo XIX dirigida por Eugen Graetzer que se mantuvo en activo entre 1891 y 1908, tenía varias notas distintivas propias: la editaría una Fundación, con sede en Ámsterdam, cubriría temáticamente todas las áreas de la medicina, a cada una de las cuales se dedicaría una serie, prestaría especial atención a la producción europea, e incluiría amplios resúmenes analíticos del contenido de los artículos seleccionados en lengua inglesa, independiente del idioma en el que hubiera sido editado el artículo original, pues sus creadores tenían claro que Alemania perdería la Guerra y que la tradición germana de los repertorios de resúmenes, encarnada en los prestigiosos Zentralblätter, desparecería tras el fin del conflicto. Landsberg y Freud fueron los dos primeros europeos continentales que comprendieron que el inglés sería la lingua franca de la ciencia del futuro, ese idioma universal por el que se había discutido y suspirado desde finales del siglo XIX (Andriesse, 2008, p. 145-154), y sobre el cual algunos alemanes de entonces ya habían estudiado esa posibilidad, como W. Brackebusch en 1868, en su tesis doctoral en filosofía defendida en la Universidad de Göttingen.

El creciente nacionalismo científico, notable tras la Gran Guerra, se tradujo, además, en una nueva política documental que, después de la II Guerra Mundial, se materializó en la creación de centros nacionales de información y documentación científicas ${ }^{7}$. Salvo algunas producciones muy concretas que mantuvieron su idea de universalismo, la mayoría de los países entraron en competencia produciendo repertorios propios que, básicamente, iban a recoger con especial cuidado la producción bibliográfica corriente de cada nación, ya fuera en ciencias o humanidades.

Desde el punto de vista editorial el modelo prebélico que antes señalé fue desapareciendo para ser sustituido por otro en el que grandes grupos editoriales (Elsevier, 
Springer Verlag...) han ido absorbiendo otras empresas menos potentes económicamente pero de alta calidad, así como centros documentales, repertorios y publicaciones aisladas ${ }^{8}$. Bien es cierto que aún perviven librerías comerciales independientes que no forman parte de momento de los grandes cárteles (Karger, Dekker...). El modelo académico prácticamente es inexistente, aunque aún persisten grupos, como el inglés The Company of Biologists Ltd. (Skaer, 2003, p. 117-125).

La globalización ha desbancado al internacionalismo, pues ha hecho realidad una de sus aspiraciones, la universalización del acceso al conocimiento. Pero también la globalización contiene elementos retóricos, al igual que mostré en el caso del internacionalismo. Los grandes cárteles y editoriales condicionan y limitan el acceso a la información, aunque es cierto que Internet ha hecho posible una mayor y mejor difusión informativa.

La historia de la National Library of Medicine de los Estados Unidos (NLM) sintetiza perfectamente el paso del internacionalismo a la globalización científicas, de una cultura del papel -recordemos a título de ejemplo dos empresas de John Shaw Billings, su creador, el Index Catalogue (1880-1961), hoy día imprescindible para cualquier investigación histórico-médica, y el Index Medicus que inició su aventura en 1879 y que dejó de imprimirse en 2004- a otra en la que el acceso on line a la información se ha convertido en imprescindible. En este desarrollo la $N L M$ fue pionera. Como es sabido, en 1964 lanzó MEDLARS, de uso restringido, y siete años después amplió su acceso con MEDLINE, que a partir de 1997 fue de consulta universal y gratuita a través de Internet (Blake, 1980; Miles, 1982; Greenberg y Gallagher, 2009; Bourne y Hahn, 2003, p. 219223 y 289-295).

\section{NOTAS}

\footnotetext{
1 "Il n'existe pas une Chimie allemande, une Chimie russe, une Chimie japonaise, il n'y a que LA CHIMIE dont les lois éprouvées sont vraies dans un pays bien que dans un autre" (Reinsach, Paul S., Introduction, en: Eijkman, 1911, s. p.). Las mayúsculas, en el original.

2 La explosión de las patentes, como mostró Hulme en 1923, se dio a partir de 1870, y su crecimiento fue espectacular. Si en 1850 apenas se registraron 500 inventos, en 1870 fueron 9.500 y en 1921 más de 18.000 (Hulme, 1923).

3 En 1938 Wells publicó Brain World, un conjunto de ensayos en los que incorporó buena parte de las ideas de Otlet y Ostwald sobre el tema (Rayward, 1999).

${ }^{4}$ En la primavera de 1935 se celebró en España el II Congreso Internacional de Bibliotecas y Bibliografía. Las Actas del mismo no se editaron hasta 1949, por instancia de la Federación Internacional de Asociaciones de Bibliotecarios (Actas y Trabajos ..., 1949). Entre otros temas se abordó el Préstamo Internacional, las Bibliotecas Populares, las Bibliotecas Especiales, etc. Se ha señalado que buena parte de los temas tratados no concluyeron en acciones concretas. Además un número significativo de ponencias presentadas al mismo nunca se editaron (Domínguez Sánchez y Domínguez Sanjurjo, 1993).

5 <http://www.nypl.org/help/about-nypl/history>. [Consulta: 15 de abril de 2011].

6 Ernest Solvay (1838-1922) era un químico e industrial belga, que dedicó parte de sus ganancias a fines filantrópicos. Además de fundar un Institut des Sciences Sociales (1894), adscrito a la Universidad de Bruselas, creó una escuela de negocios y patrocinó las célebres conferencias Solvay de Física y de Química.
} 
7 Algunos ejemplos de centros estatales son: en 1939 se fundó el CNRS francés; en 1952 el VINITI soviético. En 1953 el CID, adscrito al CSIC en Madrid, que en 1975 se convertiría en el Centro Nacional de Documentación Científica; en Frankfurt en 1961 nace el Institut für Dokumentationwesen. Frente a este modelo estatal, en el mundo anglosajón predominaron los abiertos por la iniciativa privada, no jerarquizados, con sucursales en función de la evolución de los mercados, y que eran editores además de repertorios universalistas, algunos de ellos especializados. El caso más paradigmático es el Institute of Scientific Information de Eugen Garfield (1958).

${ }^{8}$ Por ejemplo, el grupo editorial DOYMA, editor de prestigiosas revistas españolas, que fue adquirido en 2005 por Elsevier, o el ISI de Garfield, que en 1992 pasó a manos de Thomson Scientific and Health Care. La importante revista Nature hoy forma parte del grupo Macmillan Publishers, y Lancet es propiedad de Elsevier. En 1971 Elsevier adquirió Excerpta Medica, con lo cual dejó de editarse por la Excerpta Medica Foundation. En la primavera de 2011, finalmente, Elsevier adquirió tras arduas negociaciones el portal médico español Fisterra.

\section{BIBLIOGRAFÍA}

Actas y trabajos del II Congreso Internacional de Bibliotecas y Bibliografía. 2 vol., Madrid: Asociación de Bibliotecarios y Bibliógrafos de España, 1949.

ALDERSON, B. Andrew Carnegie. The Man and His Work. Doubleday: Page \& Co., 1902.

ANDREWS, S.P. The Primary Synopsis of Universology and Alwato, the New Scientific Universal Language. New York: Dion Thomas, 1871.

ANDRIESSE, C.D. Dutch Messengers. A History of Science Publishing, 1930-1980. Leiden-Boston: Koninklijke Brill, NV, 2008.

BECK, H. Die internationale bibliographie und ihre zukunft. Dresden: Boehmert, 1907.

BLAKE, J.B. (ed.). Centennial of Index Medicus (1879-1899). Bethesda: National Library of Medicine, 1980.

BOURNE, C.P. y HAHN, T.B. A History of Online Information Services, 1963-1976. Cambridge-London: MIT Press, 2003.

BRACKEBUSCH, W. Is English destined to become the Universal Language of the World? An inaugural dissertation for obtaining the Degree of Doctor of Philosophy in the University of Göttingen. Göttingen: The University Press, 1868.

BROWN, R.E. Rockefeller Medicine Men. Medicine and Capitalism in America. Berkeley-Los Angeles-London: University of California Press, 1979.

BÜHRER, K.W. Raumnot und Weltformat. München: Die Brücke, 1912.

BÜHRER, K.W. y SAAGER, A. Die Welt Registratur. Das Melvil-Deweysche Dezimal System. München: Die Brücke, 1912.

BURCHARDT, L. Technischer Fortschritt und Wandel: Das Beispiel der Taylorismus. En TREUE, W. (ed.). Deutsche Technikgeschichte. Göttingen: Vandenhöck \& Ruprecht, 1997.

CHANARON, J. y PERRIN, J. Science, Technology and Work Organization. International Journal of Technology Management, 1987, vol. 2, p. 377-389.

COOK, A. Academic Publications before 1940. En FREDRIKSSON, E.E. (ed.). A Century of Science Publishing. A Collection of Essays. Amsterdam: IOS Press, 2003, p. $15-24$. 
CRAWFORD, E. Nationalism and Internationalism in Science, 1880-1939. Cambridge: CUP, 1992.

DOMÍNGUEZ SÁNCHEZ, P. y DOMÍNGUEZ SANJURJO, M. R. II Congreso Internacional de Bibliotecas y Bibliografía (año 1935): Una aproximación. Boletín de la ANABAD, 1993, vol. 43, p. 41-54.

DUBRAVSKY, J.V. Fritz Haber: ammonia synthesis, poison gas and the Great War. Chemistry in Australia, 2008, vol. 4, p. 4-7.

EDWARDS, P.N. Beyond the Ivory Tower. "A Vast Machine": Standards as Social Technology. Science, 2004, vol. 304, p. 826-828.

EIJKMAN, P.H. L'Internationalisme Médical. Amsterdam: F. van Rosse, 1910; L'Internationalisme Scientifique. Sciences Pures et Lettres. La Haye: W. P. van Stockum et Fils, 1911.

FARKAS-CONN, I.S. From Documentation to Information Science. New York-Westport: Greenwood Press, 1990.

GIUNCHEDI, C. y GRIGNANI, E. La Società Bibliografica Italiana. 1896-1915. Firenze: Leo S. Olschki, 1994.

GREENBERG, S.J. y GALLAGHER, P.A. Index Medicus, Index-Catalogue, and IndexCat. J. Med. Libr. Assoc., 2009, vol. 97, p. 108-113.

GUÉRARD, A.L. A Short History of the International Language Movement. New York: Boni and Liveright, 1921.

HAMPSON, G. A Classification of the Pyralidae, subfamily Hypsotropinae. Proc. Roy. Zool. Soc. London, Parts I \& II, 1918, p. 55-131.

Handbook of the New York Public Library. New York: The New York Public Library, 1916.

HAPKE, T. From the World Brain to the First Transatlantic Information Dialogue: activities in information and documentation in Germany in the first half of the 20th Century. IFLA Journal, 2003, vol. 29, p. 364-377; Ordnung, Fragmentierung und Popularisierung: Wilhelm Ostwald zur wissenschaftlichen Information und Kommunikation. En KRUG, K. (ed.). Wissenschaftstheorie und -organisation: Vorträge zu dem Symposium anlässlich des 150. Geburtstages von Wilhelm Ostwald am 18. September 2003. Großbothen: Wilhelm-Ostwald-Gesellschaft, 2004, p. 63-78.

HJØRLAND, B. Empiricism, rationalism and positivism in library and information science. J. Doc., 2005, vol. 61, p. 130-155.

HULME, E.W. Statistical Bibliography in Relation to the Growth of Modern Civilization. London: Butler \& Tanner Grafton \& Co., 1923.

International Exchange List of the Smithsonian Institution. Corrected to September, 1903. Washington: Smithsonian Institution, 1904.

JAHR, T. y STROHM, A.J. (1902). Bibliography of Cooperative Cataloguing Bibliography and the Universal Catalogue (1850-1902). En Report of the Librarian of Congress. 1902. Washington: Government Printing Office, p. 111-228.

JOUOT, C. Le Congrès mondial de la documentation universelle, Paris, 1937. Paris: Université de Paris I, 1997.

LYONS, H. The Royal Society. 1660-1940. New York: Greenwood Press, 1968. 
MACCORMICK, F. How Terms of Peace can be automatically prepared while the War is still going on. Zurich: Concilium Bibliographicum, 1916; Via Pacis: Further Considerations concerning "How Terms of Peace can be automatically Prepared While the War is still Going On. Zurich: Concilium Bibliographicum, 1917.

MEHRA, J. The Solvay Conference on Physics. Aspects of the Development of Physics since 1911. Dordrecht-Boston: D. Reidel Publishing Co, 1975.

MILES, W.D. A History of the National Library of Medicine. The Nation's Treasury of Medical Knowledge. Bethesda: US Department of Health and Human Services, 1985.

NEUMANN, A.A. Cyrus Adler. A Biographical Sketch. American Jewish Year Book, 1940-1941, vol. 42, p. 23-145.

NICAULT, C. Julien Cain (1887-1974). Revue pour l'histoire du CNRS, 2005, vol. 12 <http://histoire-cnrs.revues.org/1339?\&id=1339>. [Consulta: 3 de mayo de 2011].

OLAGÜEE DE ROS, G.; ASTRAIN GALLART, M.; MENENDEZ NAVARRO, A. y MEDINA DOMENECH, R. La introducción de la Clasificación Decimal Universal en España, 1896-1923. En Carrillo, J. L. y Olagüe, G. (Ed). Actas del XXXIII Congreso Internacional de Historia de la Medicina. Granada-Sevilla, 1 a 6 de septiembre de 1992. Sevilla: Imprenta Pinelo, 1994, p. 1041-1056.

OLAGÜE DE ROS, G. et alia. Internacionalismo y ciencia. Las bases sociocientíficas del movimiento documental europeo. Dynamis, 1997, vol. 17, p. 317-340.

OLAGÜE DE ROS, G. e IGNACIUK, A. Nacionalismo e internacionalismo en ciencia. A propósito de cinco iniciativas bibliográficas nacionales surgidas al amparo del International Catalogue of Scientific Literature (1901-1921). En Porras Gallo M. I., Gutiérrez Rodilla, B., Ayarzagüena Sanz, M. y De las Heras Salord, J. (Ed). Transmisión del conocimiento médico e internacionalización de las prácticas sanitarias: una reflexión histórica. XV Congreso de la Sociedad española de Historia de la Medicina Ciudad Real, 15-18 de junio de 2011. Ciudad Real: SEHM-Facultad de Medicina de la Universidad de Castilla-La Mancha, 2011, p. 99-103.

OSTWALD, W. Energetische Grundlagen der Kulturwissenschaft. Leipzig: Klinkhardt, 1909; Das Gehirn der Welt. München: Die Brücke, 1912; Scientific management for scientists. "The Bridge". The trust idea applied to intellectual production. Sci. Amer., 1913, vol. 108, p. 5-6.

QUÉRÉE, H.C. Library Fittings. En MIDDLETON, G.A.T. (ed.). Modern Buildings, Their Planning, Construction and Equipment. Vol. VI, London: Caxton Publ. Co., 1905, p. 58-67.

RAYWARD, W.B. The Universe of Information. The Work of Paul Otlet for Documentation and International Organisation. Moscow: FID-VINITI, 1975; The International Exposition and the World Documentation Congress, Paris 1937. Libre Quart, 1983, vol. 53, p. 254-268; H. G. Wells's Idea of a World Brain: A Critical Reassessment. Journal of the American Society for Information Science, 1999, vol. 50, p. 557-573.

RICHARDS, P.S. Scientific Information in Wartime. The Allied-Germany Rivalry, 19391945. Westport-London: Greenwood Press, 1994.

RODRÍGUEZ OCAÑA, E. Foreign Expertise, Political Pragmatism and Professional Elite: The Rockefeller Foundation in Spain, 1919-39. Studies in the History and Philosophy of Biological and Biomedical Sciencies, 2000, vol. 31, p. 447-461. 
ROVIRA, T. La xarxa catalana de biblioteques populars: dels orígens a la desfeta de 1939. Item, 1994, no 14, p. 5-20.

SACHSSE, R. Das Gehirn der Welt: 1912. Telepolis, $1998<\mathrm{http}: / / w w w . h e i s e . d e / t p / r 4 /$ artikel/2/2481/1.html>. [Consulta: 4 de marzo de 2011].

SAGREDO FERNÁNDEZ, F. La documentación y el nacimiento de las Naciones Unidas. Scire, 2004, p. 21-30.

SALOMON, J. J. The Internationale of Science. Scientific Studies, 1971, vol. 1, p. 23-42.

SCHROEDER-GUDEHUS, B. Challenge to Transnational Loyalties: International Scientific Organizations after the First World War. Sci. Stud., 1973, vol. 3, p. 93-118.

SHERA, J. H. y CLEVELAND, D.B. History and Foundations of Information Science. Ann. Rev. Info. Sci. Technol., 1977, vol. 12, p. 249-275.

SKAER, R. Scientists as Publishers: The Company of Biologists Ltd. En FREDRIKSSON E.H. (ed.), op cit., 2003, p. 117-125.

SOMSEN, G.J. A History of Universalism: Conceptions of the Internationality of Science from the Enlightenment to the Cold War. Minerva, 2008, vol. 46, p. 361-379.

TATE, V.D. Introducing American Documentation. A Quaterly Review of Ideas, Techniques, Problems and Achievements in Documentation. American Documentation, 1950, vol. 1, p. 3-7.

Treaty concerning the formation of a General Postal Union signed at Berne, october 9, 1874. Washington: Government Printing Office, 1875.

WARD, H.B. Herbert Haviland Field. Science, 1921, vol. 54, p. 424-428. 